\title{
Exophthalmos associated to orbital zygomatic mucocele and complex maxillary malformation in a puppy
}

\author{
Alessandro Cirla ${ }^{1,2,{ }^{*}}$, Marco Rondena ${ }^{3}$, Giovanna Bertolini ${ }^{1}$ and Giovanni Barsotti ${ }^{2}$ \\ ${ }^{1}$ San Marco Veterinary Clinic, via Sorio 114/c - 35141 Padova, Italy \\ ${ }^{2}$ Department of Veterinary Science, University of Pisa, via Livornese Lato Monte - 56124 San Piero a Grado, Pisa, \\ Italy \\ ${ }^{3}$ San Marco Veterinary Laboratory, via Sorio 114/c - 35141 Padova, Italy
}

\begin{abstract}
A case of exophthalmos due to zygomatic mucocele in a puppy with ipsilateral segmental maxillary atrophy is reported. A 7-month-old, mixed breed, male dog suffered the sudden-onset of unilateral painful exophthalmos and a gradual swelling of the right temporal region. A compressing, right retrobulbar mass was observed by ultrasound. Computed tomography revealed a large multiloculated cyst-like lesion of the right zygomatic gland projecting into the orbital space, thus displacing the eyeball. The ipsilateral molar part of the maxillary bone was underdeveloped, besides showing crowded, abnormal, multiple, unerupted maxillary molar teeth in the caudal maxillary region. Modified lateral orbitotomy and a selective caudal maxillary bone access were performed. The cyst-like lesion was removed and the zygomatic gland and the wall was collected for histology, which confirmed the mucocele. Clinical and imaging examinations six months after surgery showed neither recurrence of the mucocele nor ocular abnormalities. A possible common pathogenic mechanism involving these two conditions could be hypothesized. Keywords: Computed tomography, Dog, Exophthalmos, Maxillary atrophy, Zygomatic mucocele.
\end{abstract}

\section{Introduction}

The major salivary glands of the dog are the parotid, sublingual, mandibular and zygomatic glands. The zygomatic gland, in comparison with other salivary glands, is the least frequently involved in pathological conditions in dogs (Schmidt and Betts, 1978; Bellenger and Simpson, 1992; Cannon et al., 2011; Nemec et al., 2015). Salivary gland mucocele is a collection of saliva in a cavity lined by granulation tissue that occurs following disruption of a salivary gland or its ducts. The mucocele is the most common pathological condition of the salivary glands of the dog, most often involving the sublingual gland, although it can involve the other glands (Karbe and Nielsen, 1966; Bellenger and Simpson, 1992). Zygomatic mucocele is a condition rarely observed in veterinary medicine (Miller and Pickett, 1989; Bellenger and Simpson, 1992; Speakman et al., 1997; McGill et al., 2009), but it should be considered as a possible diagnosis in dogs presenting exophthalmos, protrusion of the third eyelid and a fluctuant, palpable, soft tissue mass around the zygomatic bone (Noghreyan et al., 1996). Since the zygomatic gland is located ventrally to the zygomatic arch, mild changes may not be detected in the early stages of the disease. Furthermore, clinical signs such as exophthalmos may be similar to the ones of other orbital diseases. Unilateral exophthalmos in dogs is observed in conjunction with a variety of diseases. The possible differential diagnoses for exophthalmos include primary or secondary orbital neoplasia, inflammatory orbital diseases, orbital varices, and cysticlexudative orbital diseases (zygomatic salivary gland mucocele, hematic cyst secondary to trauma or immune-mediated disease, dacryops, or orbital oedema) (Karbe and Nielsen, 1966; Harvey, 1971; Bellenger and Simpson, 1992; Guinan et al., 2007; Atkins et al., 2010; Adams et al., 2011; Cannon et al., 2011; Philp et al., 2012). Despite the variable underlying causes, the clinical signs of these various conditions are very similar to one another. Diagnostic imaging can be useful to differentiate a zygomatic gland disease from other orbital diseases (Penninck et al., 2001; Bartoe et al., 2007; Boston, 2010; Lee et al., 2014). Cases of zygomatic mucocele were reported in a cat and in a ferret (Miller and Pickett, 1989; Speakman et al., 1997).

The present report concerns the diagnostic and therapeutic management of a complex maxillary malformation and concurrent zygomatic mucocele in a puppy. To the authors' best knowledge, this is the first report on this condition in a dog

\section{Case details}

A 7-month-old, mongrel male dog was brought to our attention for further evaluation of an acute, painful, rapidly progressive enlargement of the right eye (OD). The dog's general condition was good, but facial 
oedema was evident, mainly involving the temporal, frontal and zygomatic regions, as well as discomfort when opening the mouth. No palpable regional lymph nodes were detected. The dog's medical history was unremarkable, no history of trauma or previous ocular diseases were reported. Ophthalmic examination of the right eye revealed ocular discomfort, mucous secretion, protrusion of the nictitating membrane, chemosis and congestion of conjunctival vessels, moderate exophthalmos and lagophtalmos. The globe was substantially resistant to retropulsion, no visibile buccal lesions were detected. Complete ophthalmic examination of the OD was compromised by the nictitating membrane protrusion. However, the limited examination did not show any ocular lesions (Fig.1). No abnormalities were observed in the left eye.

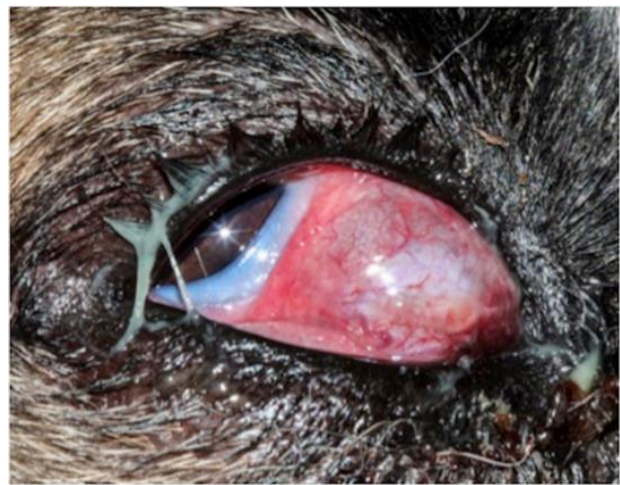

Fig. 1. Clinical appearance of OD. Note the mucous secretion, protrusion of the nictitating membrane, chemosis and congestion of conjunctival vessels.

A compressive orbital space-occupying lesion was clinically suspected and confirmed by ultrasonography (Logiq C5 Premium, $10 \mathrm{MHz}$ linear probe, GE Healthcare). Orbital ultrasonographic examination revealed a 3,5 x 1,7 cm round echogenic structure with a large volume of anechoic content. Ocular ultrasound was normal (Fig.2).

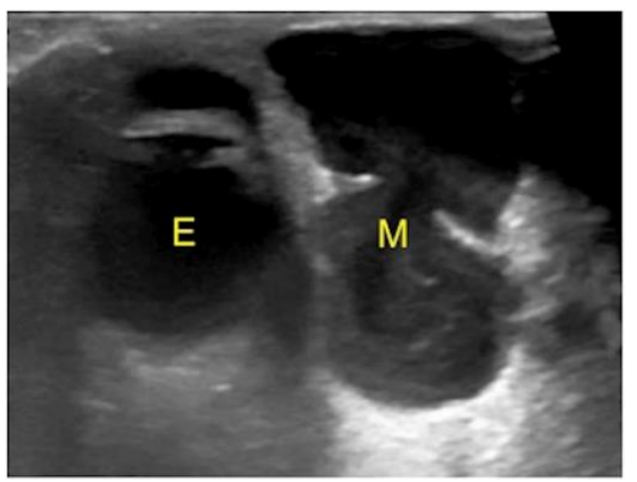

Fig. 2. Orbital ultrasound (10 MHz linear probe, transverse scan, temporal fossa approach) showing a large orbital fluidfilled space-occupying mass (M). The OD ocular examination (E) showed no abnormalities.
Ultrasound-guided fine-needle aspiration revealed a clear watery to viscous fluid highly suggestive of saliva collection.

Results of CBC analyses showed moderate leukocytosis (white blood cells: 26000/ $\mu$; reference interval 5450/ $\mu \mathrm{l}-12980 / \mu \mathrm{l})$. Serum biochemistry, coagulation test and urinalysis were unremarkable.

Computed tomography scan (CT) was scheduled to better define the extent and the origin of the lesion. After intramuscular premedication with $0.2 \mathrm{mg} / \mathrm{kg}$ methadone (Semfortan, Dechra Pharmaceuticals) and 2 $\mu \mathrm{g} / \mathrm{kg}$ dexmedetomidine (Dexdomitor, Orino Pharma), general anesthesia was induced with $4 \mathrm{mg} / \mathrm{kg}$ propofol (Vetofol, Esteve) intravenously and maintained with isofluorane (IsoFlo; Halocarbon Laboratories) in oxygen. A 16 GA intravenous catheter was placed in the right cephalic vein for fluid administration throughout the anestethic period.

Computed tomography was performed using a secondgeneration dual-source CT scanner (SOMATOM Definition Flash, Siemens Healthcare, Milan Italy) with following parameters: single source, spiral mode, pitch $0.5,128 \times 0.6 \mathrm{~mm}$ detector configuration, $120 \mathrm{KVp}$ and $200 \mathrm{mAs}$ and double reconstruction kernel for bone (H70) and soft tissue (B30) with $0.3 \mathrm{~mm}$ reconstruction interval.

Four CT series were obtained: a pre-contrast series, followed by two post-contrast series, in arterial and venous phase. Finally, a third late post-contrast series was obtained. Contrast medium (iodixanol $320 \mathrm{mgI} / \mathrm{mL}$ ) was injected at $37^{\circ} \mathrm{C}$ into a cephalic vein $(640 \mathrm{mgI} / \mathrm{kg}$ ) through the IV catheter, at $3,5 \mathrm{~mL} / \mathrm{s}$ injection rate, followed by a saline flush with same injection rate via a dual-syringe injector system (Medrad, Stellant CT Injection System, Bayer, Milan, Italy).

CT images revealed a large multiloculated cyst-like lesion in the right orbital space, involving the zygomatic gland and displacing the eyeball dorsolaterally. The lesion had a thin wall and contained homogeneous, non-enhancing fluid. The molar part of the ipsilateral maxillary bone was underdeveloped. Crowded, abnormal, multiple, unerupted maxillary molar teeth were identified in the caudal maxillary region (Fig.3).

Differential diagnosis included odontogenic cyst (i.e. dentigerous cyst), maxillary bone cysts, and zygomatic mucocele associated to a maxillary bone defect.

Surgical exploration of the mass was recommended, with a view to its complete excision.

Modified lateral orbitotomy and selective caudal maxillary bone access were performed to provide ventral exposure of the orbit and dental extraction, while minimizing trauma to the globe and supporting structures. 


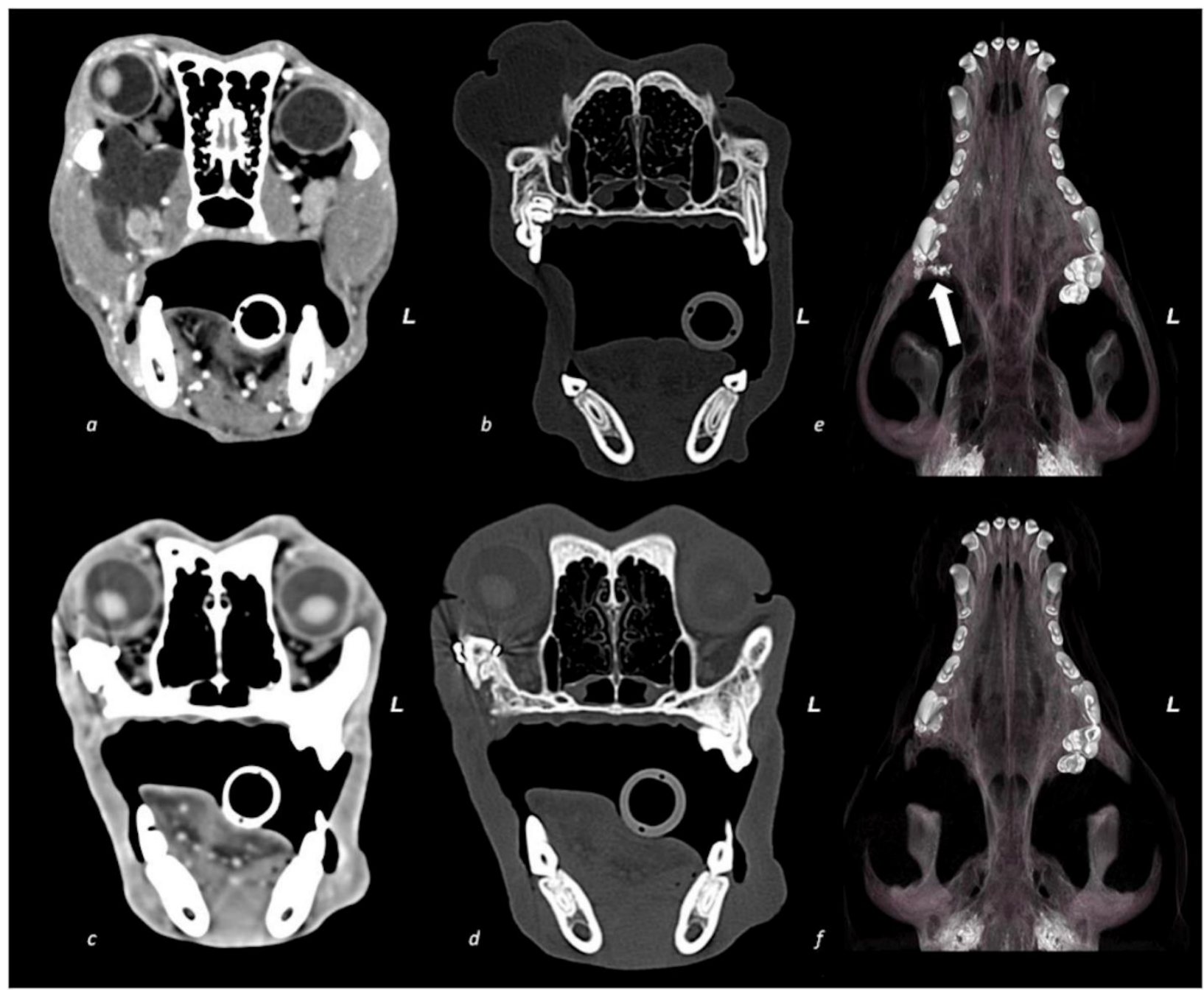

Fig. 3. Transverse plane through the orbit showing a large mass displacing the eyeball dorso-laterally $(\boldsymbol{a})$ and crowded, multiple, abnormal, unerupted maxillary molars $(\boldsymbol{b})$. Volume rendering, ventral view $(\boldsymbol{e})$. Note the underdeveloped molar part of the right maxillary bone (arrow) and crowded, multiple, abnormal, unerupted maxillary molar teeth. Six month follow-up CT exam: transverse plane through the orbit $(\boldsymbol{c}, \boldsymbol{d})$ and volume rendering, ventral view showed no recurrence of the orbital pathology $(\boldsymbol{f})$.

The dog was premedicated with $0.2 \mathrm{mg} / \mathrm{kg}$ methadone (Semfortan, Dechra Pharmaceuticals) and $2 \mu \mathrm{g} / \mathrm{kg}$ dexmedetomidine (Dexdomitor, Orino Pharma) intramuscularly. Anaesthesia was induced with $5 \mathrm{mg} / \mathrm{kg}$ propofol (Vetofol, Esteve) intravenously and maintained with a mixture of isofluorane (IsoFlo; Halocarbon Laboratories) and oxygen in a closed breathing circuit. Cefazolin (Cefazolina, Teva) was administered intravenously at the dosage of $22 \mathrm{mg} / \mathrm{kg}$, 25 minutes before the anaesthetic induction. A curvilineal incision was made along the dorsal margin of the zygomatic arch. The palpebral nerve and the dorsal buccal branch of the facial nerve were identified through blunt dissection. These structures were delicately separated from the underlying tissues and retracted.

Prospective zygomatic osteotomy sites were identified, and $0.35 \mathrm{~mm}$ Kirschner wire placed in a hand-held orthopedic Jacobs chuck was used to drill sets of holes adjacent to each site.

Transection of the zygomatic arch was performed with a pneumatic saw between the previously drilled holes, and reflected dorsally without transection of the orbital ligament. The cystic structure was bluntly undermined from the surrounding tissues and its dorsal aspect was noted as connecting under the zygomatic arch to the ventral orbit. The mass and the zygomatic gland were removed as a whole and submitted for histopathological examination.

After saline irrigation, the transected zygomatic arch was replaced and fixed with a 22-gauge orthopedic wire. The surgical site was closed as normal. A posterior maxillary bone fenestration was then performed to allow the included teeth extraction. The 
bone cavity was thoroughly curetted and rinsed with sterile saline. A mucoperiosteal flap was raised buccally to close the surgical wound.

The dog recovered uneventfully from anesthesia and surgery, the exophthalmos resolved immediately postoperatively. At this time the patient received a complete ophthalmic examination of both eyes and showed no abnormalities. Complete ophthalmic examination included neuro-opthalmic examination, slit-lamp biomicroscopy and indirect ophthalmoscopy. Schirmer tear test I, retension of corneal sodium fluorescein dye and intraocular pressure estimation were performed.

The tissues submitted for histopathology were immediately fixed in 10 per cent neutral-buffered formalin, embedded in paraffin, sectioned at $4 \mu \mathrm{m}$ and stained with haematoxylin and eosin. Microscopic evaluation of the biopsy tissues revealed numerous small acini of zygomatic gland embedded in a thick granulation tissue delineated in a border by abundant fibrin and amorphous hyaline homogeneous material (salivary secretion), partially infiltrated by macrophages consistent with mucocele (Fig. 4). There was no evidence of infection or malignant changes. Postoperatively, an Elizabethan collar prevented selftrauma at the surgical site.

The dog was placed on cephalexin orally at the dose of $22 \mathrm{mg} / \mathrm{kg}$ (IcfVet, Icf) for antibiotic prophylaxis. Methadone (Semfortan, Dechra) was given intravenously for the first 48 hours at the dose of 0.2 $\mathrm{mg} / \mathrm{kg}$ for surgical pain management, and carprofen (Rimadyl, Pfizer Animal Health) was prescribed at a dose of $2.2 \mathrm{mg} / \mathrm{kg}$ to address inflammation.
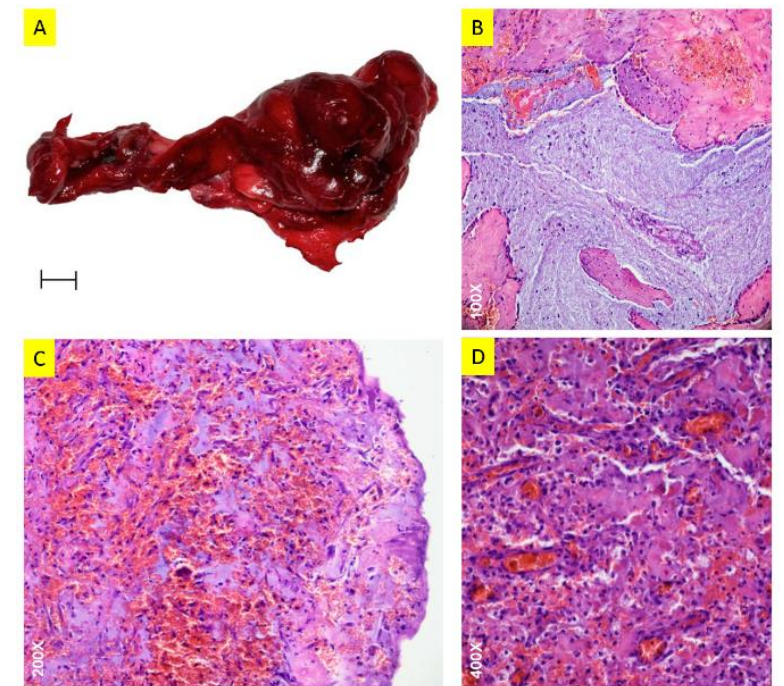

Fig. 4. Gross anatomy of the mass (A). Histopathology of the mass haematoxylin and eosin x100 (B), x200 (C) and x400 (D). Note the granulation tissue embedding large, irregularly shaped lakes of clear amorphous material (mucous secretion/salivary secretion of zygomatic gland origin).
The dog was discharged from the hospital three days after surgery and re-evaluated 10 days postoperatively, at which time the skin sutures were removed. The incision was healing appropriately and there was no sign of orbital or periocular swelling. At this time, oral examination showed no abnormalities and the dog was eating well. No clinical signs of ocular complications secondary to the exophthalmia such as exposure keratitis or fundus lesions were detected. No orbital recurrence of the mucocele was present. At the 6-month clinical follow-up, the CT exam showed no recurrence (Fig. 3).

Discussion
We described a complex case of exophthalmos due to a zygomatic mucocele in a puppy with ipsilateral segmental maxillary malformation. A possible relationship between these two conditions could be hypothesized on the basis of advanced imaging features. Advanced imaging modalities play a key role in identifying the cause of exophthalmos. Both CT and MR imaging have been reported as useful diagnostic tools for the evaluation of canine mucocele (Boland $e t$ al., 2013; Torad and Hassan, 2013; Namsoon et al., 2014; Nemec et al., 2015).

Neoplasia, sialoliths, trauma, and foreign bodies invading the glandular structures have been identified as possible causes of mucocele in dogs (Harvey, 1971; Guinan et al., 2007; Atkins et al., 2010; Adams et al., 2011; Philp et al., 2012). Zygomatic mucocele in dogs has also been described as a postoperative complication secondary to caudal hemimaxillectomy or maxillary tooth extraction (Clarke and L'Eplattenier, 2010; Adams et al., 2011).

The puppy of the present report had no history of previous maxillary surgery or dental procedures. Furthermore in the present case, a trauma was not reported in the medical history and the CT examination allowed to exclude the presence of sialoliths and zygomatic foreign bodies.

The distinctive feature of our case was the concomitant malformation of the maxillary bone, lining the lateral part of the cystic formation. A maxillary epithelial cyst expanding into the orbital space was reported in a dog (Featherstone and Llabres Diaz, 2003) and was initially suspected also in the present case. Brachygnathia and other maxillary bone developmental defects were reported alone or in association with other craniofacial anomalies in dogs (Duncan et al., 1994; Nemec et al., 2015).

In humans, maxillary hypoplasia involving the dentoalveolar area is described as a complex disease associated to basal deformity (Jackson, 1989; Mueller and Callanan, 2007; Rege et al., 2012). This anomaly has been also described in a rare congenital disorder known as Crouzon syndrome (Padmanabhan et al., 2011). 
The maxillary congenital hypoplasia somehow associated with the zygomatic mucocele cannot be ruled out. In general, the zygomatic mucocele clinically behaves as a progressively space-occupying lesion that can cause distortion of the surrounding bone (Mould, 1990; Featherstone and Llabres Diaz, 2003).

The focal, circular depression of the maxilla is uncommon for a benign lesion, although such presumed pressure necrosis of the bone has been previously described (Mould, 1990; Featherstone and Llabres Diaz, 2003). It is not unreasonable to assume that an expanding zygomatic mucocele might behave in a similar manner. In our case, the zygomatic mucocele might have created progressive pressure atrophy, causing the depression of the maxilla and its underdevelopment.

The combination of accurate clinical examination, interpretation of advanced cross-sectional imaging, including CT, and combined surgery was successful for the treatment of the zygomatic mucocele in our case associated to a maxillary malformation. Early diagnosis of the disease and its surgical management prevented later ocular complications.

\section{Conflict of interest}

The authors declare that there is no conflict of interests.

\section{References}

Adams, P., Halfacree, Z.J., Lamb, C.R., Smith, K.C. and Baines, S.J. 2011. Zygomatic salivary mucocoele in a Lhasa apso following maxillary tooth extraction. Vet. Rec. 168, 458-460.

Atkins, R.M., Hecht, S., Westermeyer, H.D. and McLean, N.J. 2010. What Is Your Diagnosis? J. Am. Vet. Med. Assoc. 237, 1375-1376.

Bartoe, J.T., Brightman, A.H. and Davidson, H.J. 2007. Modified lateral orbitotomy for vision-sparing excision of a zygomatic mucocele in a dog. Vet. Ophthalmol. 10, 127-131.

Bellenger, C.R. and Simpson, D.J. 1992. Canine sialocoeles - 60 clinical cases. J. Small Anim. Pract. 33, 376-380.

Boland, L., Gomes, E., Payen, G., Bouvy, B. and Poncet, C. 2013. Zygomatic salivary gland diseases in the dog: three cases diagnosed by MRI. J. Am. Anim. Hosp. Assoc. 49, 333-337.

Boston, S.E. 2010. Craniectomy and orbitectomy in dogs and cats. Can. Vet. J. 51, 537-540.

Cannon, M.S., Paglia, D., Zwingenberger, A.L., Boroffka, S.A., Hollingsworth, S.R. and Wisner, E.R. 2011. Clinical and diagnostic imaging findings in dogs with zygomatic sialadenitis: 11 cases (1990-2009). J. Am. Vet. Med. Assoc. 239, 12111218.

Clarke, B.S. and L'Eplattenier, H.F. 2010. Zygomatic salivary mucocoele as a postoperative complication following caudal hemimaxillectomy in a dog. J. Small Anim. Pract. 51, 495-498.

Duncan, W.K., Silberman, S.L., Trubman, A. and Meydrech, E.F. 1994. Prevalence and racial distribution of primary canine hypoplasia of the maxillary canine. Ped. Dent. 16, 365-367.

Featherstone, H. and Llabres Diaz, F. 2003. Maxillary bone epithelial cyst in a dog. J. Small Anim. Pract. 44, 541-545.

Guinan, J., Willis, A.M., Cullen, C.L. and Walshaw, R. 2007. Postenucleation orbital sialocele in a dog associated with prior parotid duct transposition. Vet. Ophthalmol. 10, 386-389.

Harvey, C.E. 1971. Traumatic frontal mucocoele in a dog: a case report. J. Small Anim. Pract. 12, 399403.

Jackson, I.T. 1989. Maxillary hypoplasia. Clin. Plas. Surg. 16, 757-775.

Karbe, E. and Nielsen, S.W. 1966. Canine Ranulas, Salivary Mucoceles and Branchial Cysts. J. Small Anim. Pract. 7, 625-630.

Lee, N., Choi, M., Keh, S., Kim, T., Kim, H. And Yoon, J. 2014. Zygomatic Sialolithiasis Diagnosed with Computed Tomography in a dog. J. Vet. Med. Sci. 76, 1389-1391.

McGill, S., Lester, N., McLachlan, A. and Mansfield, C. 2009. Concurrent sialocoele and necrotising sialadenitis in a dog. J. Small Anim. Pract. 50, 151156.

Miller, P.E. and Pickett, J.P. 1989. Zygomatic salivary gland mucocele in a ferret. J. Am. Vet. Med. Assoc. 194, 1437-1438.

Mould, J.R.B. 1990. Cholesterol granuloma of the maxilla in a dog. J. Small Anim. Pract. 31, 208-211.

Mueller, D.T. and Callanan, V.P. 2007. Congenital malformations of the oral cavity. Otolaryngol. Clin. North Am. 40, 141-160.

Namsoon, L., Mihyun, C., Seoyeon, K., Kim, T., Kim, H. and Yon, J. 2014. Zygomatic Sialolithiasis Diagnosed with Computed Tomography in a Dog. J. Vet. Med. Sci. 76, 1389-1391.

Nemec, A., Daniaux, L., Johnson, E., Peralta S. and Vertraete, F.J. 2015. Craniomaxillofacial abnormalities in dogs with congenital palatal defects: computed tomographic findings. Vet. Surg. 44, 417-422.

Noghreyan, A., Gatot, A., Hertzanu, Y. and Fliss, D.M. 1996. Zygomatic mucocele causing facial swelling. J. Oral Maxillofac. Surg. 54(12), 1469-1471.

Padmanabhan, V., Hegde, A.M. and Rai, K. 2011. Crouzon's syndrome: a review of literature and case report. Contemp. Clin. Dent. 2, 211-214.

Penninck, D., Daniel, G.B., Brawer, R. and Tidwell, A.S. 2001. Cross-sectional imaging techniques in veterinary ophthalmology. Clin. Tech. Small Anim. 
Pract. 16, 22-39.

Philp, H.S., Rhodes, M., Parry, A. and Baines, S.J. 2012. Canine zygomatic salivary mucocoele following suspected oropharyngeal penetrating stick injury. Vet. Rec. 171, 402.

Rege, I.C., Sousa, T., Leles, C. and Mendonca, E.F. 2012. Occurrence of maxillary sinus abnormalities detected by cone beam CT in asymptomatic patients. BMC Oral Health 12, 30-37.

Schmidt, G.M. and Betts, C.W. 1978. Zygomatic salivary mucoceles in the dog. J. Am. Vet. Med. Assoc. 172, 940-942.

Speakman, A.J., Baines, S.J., Williams, J.M. and Kelly, D.F. 1997. Zygomatic salivary cyst with mucocele formation in a cat. J. Small Anim. Pract. 38, 468470.

Torad, F.A. and Hassan, E.A. 2013. Clinical and ultrasonographic characteristics of salivary mucoceles in 13 dogs. Vet. Radiol. Ultrasound 54, 293-298. 